\title{
Dispositivo com Interface Vestível para a Aquisição, Processamento e Transmissão do Sinal Cardíaco em Exame de Eletrocardiograma
}

\author{
Vinícius Rodrigues Zanon, Eliel Marcos Rocha Romancini, \\ Fabrício de Oliveira Ourique, Analúcia Schiaffino Morales \\ Universidade Federal de Santa Catarina (UFSC) \\ Centro de Ciências, Tecnologias e Saúde - Departamento de Computação (DEC) \\ Curso de Engenharia de Computação \\ Araranguá, SC, Brasil \\ \{vinicius.zanon,eliel.romancini\}eposgrad.ufsc.br, \\ \{fabricio.ourique, analucia.morales\}eufsc.br
}

\begin{abstract}
The high number of wires and the lack of knowledge about the correct connection of the electrodes in the clinical practice of the electrocardiogram (ECG) exam are why this article aims to develop a device with a wearable interface for the capture of the signal heart. The results show that the cardiac signal was successfully captured, processed, and transmitted by the module with a wearable interface, tested, and validated in more than one individual. The ECG vest, proposed at the entrance interface, proved to be effective in using a reference guide in performing the electrocardiographic examination, providing comfort and convenience to the patient.
\end{abstract}

Resumo. A elevada quantidade de fios e o desconhecimento sobre a conexão correta dos eletrodos na prática clínica do exame de eletrocardiograma (ECG) são razões pelas quais este artigo visa o desenvolvimento de um dispositivo com interface vestível para a captação do sinal cardíaco. Os resultados mostram que o sinal cardíaco foi captado, processado e transmitido com sucesso pelo módulo com interface vestível, sendo testado e validado em mais de um indivíduo. $O$ colete ECG, proposto na interface de entrada, se mostrou eficaz na estratégia de uso como guia de referência na efetivação do exame de eletrocardiograma, apresentando conforto e comodidade ao paciente.

\section{Introdução}

Segundo a Sociedade Brasileira de Cardiologia (SBC), doenças cardiovasculares são as principais causas de morte no mundo. Estima-se que até o final do ano, 400 mil pessoas morrerão com diagnóstico referente às enfermidades cardíacas, representando $30 \%$ do total de óbitos no Brasil [SBC 2021]. Além disso, as sequelas deixadas pela pandemia atual aos milhões de infectados, poderão ser permanentes ou temporárias. Além dos danos aos pulmões, o SARS-COV-2, pode afetar o coração e outros órgãos, tais como, intestinos, sistema vascular e até mesmo o cérebro. A comunidade médica vem 
considerando a COVID-19 como uma doença sistêmica, que prefere a região pulmonar, mas pode afetar diferentes áreas do corpo, uma verdadeira agressão ao organismo [Wadman et al. 2020, Carfì et al. 2020, Landi et al. 2020]. A monitoração de pacientes através do exame de eletrocardiograma (ECG) é empregada como meio de prevenção aos riscos e avaliação de eventos cardiológicos. $\mathrm{O}$ aparelho que auxilia no diagnóstico de cardiopatias, é o eletrocardiógrafo, um equipamento de fundamental importância empregado em unidades básicas de saúde, pronto atendimentos e hospitais. O exame de ECG pode ser realizado em repouso, em movimento por esteira/bicicleta (ergométrico) ou através de Holter (monitoramento contínuo por $24 \mathrm{~h}$ em dependência domiciliar).

Embora o aparelho de ECG seja um instrumento bem consolidado na área médica, pesquisas demonstram que o procedimento metodológico sobre a execução do exame, pode comprometer o diagnóstico clínico dos pacientes [Monteiro et al. 2018, Fernandes et al. 2015]. O principal problema apresentado na literatura aponta o desconhecimento das posições corretas dos eletrodos no corpo do paciente, em sua maioria, por profissionais de saúde não médicos. No primeiro estudo $80 \%$ das conexões dos eletrodos foram colocadas em pacientes de forma errônea e, no segundo, apenas $1 \%$ acertaram. A conexão incorreta dos eletrodos pode incidir equívocos na captação e interpretação dos resultados clínicos do eletrocardiograma.

A Sociedade Brasileira de Cardiologia confere somente ao médico o poder de interpretação e inferência clínica das circunstâncias cardiológicas do paciente. Em caso de suporte clínico, o enfermeiro se faz relevante em situações emergenciais que demandam a necessidade de rápidas intervenções e tomadas de decisões [Pastore et al. 2016]. Para tanto, é necessário que ambos os profissionais executem e entendam os procedimentos do exame de forma coesa ao que são descritos nas literaturas, diretrizes, regulamentações e normas hospitalares.

Outro aspecto observado, e que se torna inconveniente na prática clínica, é a respeito da quantidade de fios do eletrocardiógrafo presos ao corpo do paciente. A elevada presença de fios, faz com que muitos auxiliares clínicos segurem os fios para que o paciente não sinta desconforto na realização do exame ou ainda não comprometa a amplitude de movimento do mesmo, essa intervenção pode influenciar no desenvolvimento da conhecida Síndrome do Jaleco Branco. A síndrome é causada pelo estresse sentido em uma consulta médica dada pela presença de profissionais de saúde, provocando um aumento pontual e temporário da pressão arterial do paciente. Estudos revelam que pacientes diagnosticados com a síndrome possuem maior propensão a ataques cardíacos, derrames, entre outros [Cohen et al. 2019]. O efeito desta síndrome pode provocar alterações eletrocardiográficas e gerar resultados com interpretações errôneas por parte de profissionais de saúde.

Sendo assim, esse artigo propõe um protótipo de interface vestível para a aquisição do sinal cardíaco, utilizando tecnologias de conexão sem fio e baixo custo (comparado com equipamentos disponíveis no mercado nacional), para a exibição do gráfico cardíaco em um monitor através da Internet. Tendo como objetivo principal, atuar no auxílio da correta execução de conexão dos eletrodos ao corpo do paciente, bem como na prática clínica do exame de eletrocardiograma. Além disso, o dispositivo também tem o propósito de eliminar a quantidade de fios conectados à base central do eletrocardiógrafo, minimizando os efeitos de acidentes técnicos presentes na realização do exame (a extensão dos fios ou soltura dos eletrodos) e diminuindo o número de diagnósticos equivocados. Ressalta-se ainda que, ao 
embarcar o dispositivo a um vestível, o mesmo fornecerá confiança metodológica para o profissional de saúde na prática clínica do exame. Tal integração exime a preocupação de conectar corretamente os eletrodos, proporcionando conforto e comodidade ao paciente na realização do exame.

O presente artigo está organizado em cinco seções. A segunda seção apresenta trabalhos correlatos em uma revisão da literatura científica sobre o tema. Na terceira seção, trata-se de materiais e métodos utilizados, validando os conceitos de desenvolvimento do protótipo através de uma visão geral que permite a compreensão de sua arquitetura e a respectiva modelagem do dispositivo vestível. Ainda é descrito a modelagem do dispositivo em um nível mais técnico enfatizando as interfaces de entrada, de processamento e de saída. Os resultados são apresentados na quarta seção. Finalmente, apresentam-se as considerações finais, a conclusão e as referências bibliográficas.

\section{Trabalhos Correlatos}

O monitoramento não invasivo para a saúde humana tem crescido nos últimos anos, diversas pesquisas para dispositivos vestíveis tem surgido nas mais variadas áreas, como por exemplo, educação, riscos profissionais, indústria, medicina e na área esportiva. Existem vários parâmetros biológicos ou biomarcadores que podem ser monitorados por sensores, muitos são projetados para usar no pulso e possuem as mais variadas funcionalidades: qualidade do sono, medição de gasto calórico, monitoração de estresse e detecção de doenças [Kamišalić et al. 2018].

Grandes empresas como a Samsung, FitBit e Oura Ring têm investido fortemente no desenvolvimento de dispositivos vestíveis, tais como, relógios e anéis capazes de rastrear digitalmente parâmetros fisiológicos cardíacos. A Apple, recentemente, lançou no mercado o primeiro smartwatch capaz de realizar o exame de eletrocardiograma com derivação única (Derivação I). O dispositivo monitora o estado de instabilidade do ritmo cardíaco, fornecendo resultados como: ritmo sinusal (batimento regular), fibrilação arterial (ritmo irregular), taquicardia (aumento do ritmo cardíaco) e bradicardia (redução do ritmo cardíaco) [Apple 2020].

Outras empresas como a Heartwear, fundada por cardiologistas da Universidade de Cambridge e MIT, anunciaram a cinta Heartsense, vestível projetado para ser usado em contato com o peito que monitora em tempo real o ritmo cardíaco contínuo, além de dispor informações sobre oximetria e temperatura corporal do paciente utilizando Inteligência Artificial para identificar as irregularidades dos parâmetros [Heartwear 2019]. A empresa GE Healthcare, desenvolveu um sistema de medição sem fio capaz de aferir, de maneira não invasiva, as frequência cardíacas do feto e da gestante, bem como, a atividade uterina. A proposta reduz a quantidade de fios ligados ao eletrocardiógrafo, propõe conforto à gestante e otimiza o fluxo de trabalho em hospitais no monitoramento cardíaco materno/fetal. O sistema é essencial em trabalho de parto e durante o acompanhamento da gestante [GE Healthcare 2019].

Outros estudos recentes têm sido dedicados ao desenvolvimento de dispositivos vestíveis que incorporam têxteis condutores para aquisição de sinais para avaliação e exploração cardíaca, neural, muscular e funções oculares [Acar et al. 2019]. Com a proposta de manufaturar eletrodos que sejam de uso confortável, os autores [Acar et al. 2018] descrevem a implementação de uma braçadeira capaz de monitorar o sinal cardíaco. Os sen- 
sores embutidos ao dispositivo são fabricados com a aplicação de nano-têxteis de grafeno, apresentando excelente conformidade na aquisição do sinal. O trabalho de [Ribeiro 2016] também propõe o desenvolvimento de eletrodos secos têxteis fabricados por filamentos condutivos de prata. A solução é suficiente para a aquisição do sinal, embora apresente mais ruídos causados pela impedância do que os eletrodos tradicionais.

O presente trabalho visa a construção de dispositivo vestível, que permita que o exame possa ser bem executado clinicamente e a distância. Buscando uma alternativa para a sua realização correta, fornecendo conforto e comodidade ao paciente durante o exame de eletrocardiograma.

\section{Materiais e Métodos}

\subsection{Arquitetura e Modelagem do Dispositivo Vestível}

Os módulos e sua integração na modelagem podem ser observados pela figura 1 que apresenta a arquitetura do vestível desenvolvido. É possível observar o seu funcionamento, através dos sensores de captação ECG embutidos ao colete, eles fornecerão dados bioelétricos de natureza analógica para o eletrocardiógrafo (interface de processamento). $\mathrm{O}$ dispositivo, uma vez ligado a uma fonte de alimentação (bateria) e composto pelos circuitos de condicionamento, aquisição e comunicação sem fio, disponibiliza a captação do sinal cardíaco a uma aplicação web. O médico (agente externo), poderá analisar, consultar e inferir cardiopatias e parâmetros vitais referentes ao sinal ECG fornecido pelo paciente no exame de eletrocardiograma. Já o agente externo (paciente) poderá, também, acompanhar em tempo real sua avaliação/exame, permitindo maior proximidade entre a execução e interpretação do exame feita pelo médico.

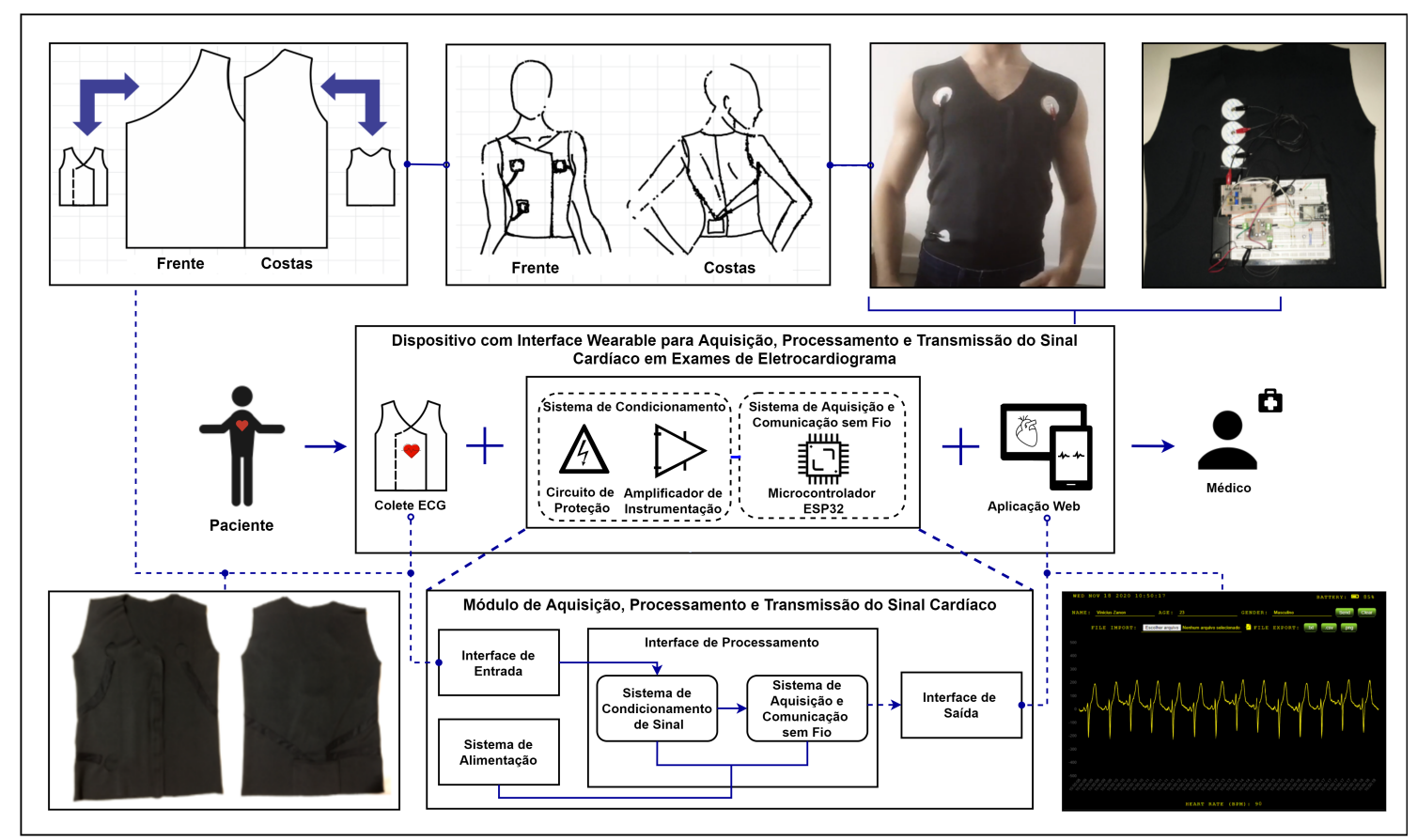

Figura 1. Visão Geral

A interface de entrada possui os sensores para a obtenção do sinal de ECG embutidos em um colete. Estes eletrodos utilizam as derivações clássicas de um eletrocardiograma, 
o qual registra a diferença de potencial entre eletrodos dadas pelas derivações de membros ou derivações precordiais. O sistema de condicionamento do sinal cardíaco é formado por um circuito de proteção referente à isolação elétrica, fornecendo ao paciente baixo risco de

choque elétrico provocado por possíveis impedâncias indesejadas. É, também, constituído pela amplificação do sinal ECG utilizando um amplificador de instrumentação com alta taxa de rejeição de modo-comum (Common-Mode Rejection Ratio - CMRR), para que o ruído, comum a ambas as entradas, seja eliminado de forma efetiva. As entradas são os sinais provenientes da ramificação direita e da ramificação esquerda, ambas referenciados ao eletrodo de referência ou terra do circuito (GND). Para que seja fornecida uma saída de qualidade, é preciso, ainda, que haja maiores cuidados quanto aos ruídos provenientes de fontes parasitas. Para a sua redução, utiliza-se técnicas de filtragem para a eliminação de radiofrequências e sobretensões.

O sistema de aquisição é responsável pelo processamento do sinal bioelétrico adquirido pelo circuito de condicionamento. O processamento é feito pelo microcontrolador ESP32, sendo constituído pela conversão do sinal analógico para digital (ADC), filtros digitais passa-baixa $(150 \mathrm{~Hz})$, passa-alta $(0.1 \mathrm{~Hz})$ e Notch $(60 \mathrm{~Hz})$. Após a etapa de aquisição, o sistema de comunicação sem fio, envia o sinal através da comunicação Wifi do ESP32 para uma aplicação Web, que pode ser acessada por qualquer dispositivo remoto (smartphones, notebooks e outros). A interface de saída tem como objetivo disponibilizar ao usuário ou agente externo, o gráfico cardíaco em tempo real disposto em uma aplicação acessada por um navegador browser onde o usuário pode realizar algumas interações com o gráfico apresentado na aplicação e verificar alguns parâmetros do dispositivo.

Por fim, para o sistema de alimentação são utilizados como fonte de energia uma bateria de $9 \mathrm{~V}$ e reguladores de tensão para fornecer as tensões de operações que a interface de processamento necessita. Para a alimentação do circuito de condicionamento fez-se o uso de um componente integrado (ICL7660S), utilizando lógica complementar, de modo a fornecer tensões de $+5 \mathrm{~V}$ e $-5 \mathrm{~V}$. A razão se dá pelo fato do circuito possuir amplificadores alimentados de forma simétrica. Já para o sistema de aquisição foi utilizada somente a carga regulada de 5V, para alimentar o microcontrolador ESP32.

\subsection{Desenvolvimento do Dispositivo com Interface Vestível}

Após a apresentação da arquitetura do dispositivo e a integração de suas funcionalidades, nesta seção serão apresentados alguns pontos com maior profundidade a respeito do desenvolvimento do vestível.

\subsubsection{Interface de Entrada}

No projeto da interface vestível foram aplicados conhecimentos e conceitos de desenho técnico, tais como, simetrias, proporções, cotas, projeções, entre outros. Pensando na maneira mais coerente de obter um bom contato entre os eletrodos e a pele, seria viável criar eletrodos têxteis com material condutivo e aumentar a pressão da roupa no corpo, para evitar o deslizamento entre a pele e os eletrodos e minimizar os artefatos de movimento do sinal. Sendo assim, após um estudo a respeito de tecnologias têxteis utilizadas em coletes, evidenciando na busca requisitos de conforto e bem-estar, foi escolhido o tecido Neoprene para a fabricação da peça. O material é um tecido emborrachado, composto por 
95\% de poliéster e 5\% de elastano. Além disso, apresenta propriedades técnicas relevantes como elasticidade (usado para diversas conformidades corporais), baixa deformação (não precisa passá-lo), propriedades isotérmicas (mantém a temperatura do corpo estável) e impermeabilidade (não absorve resíduos líquidos) [Malharia Indaial 1994]. A figura 2 apresenta o esboço e a execução da interface vestível.

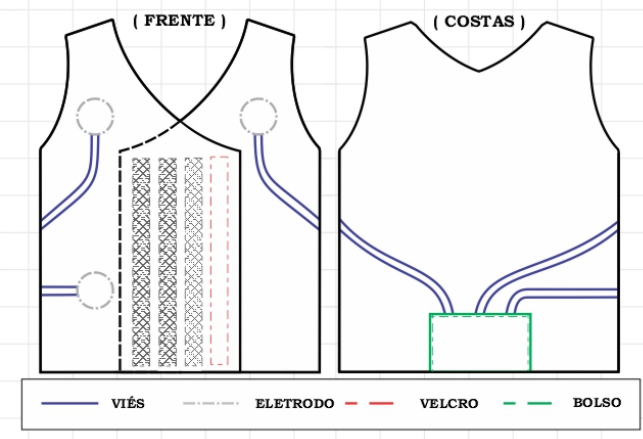

(a) Detalhamento Técnico

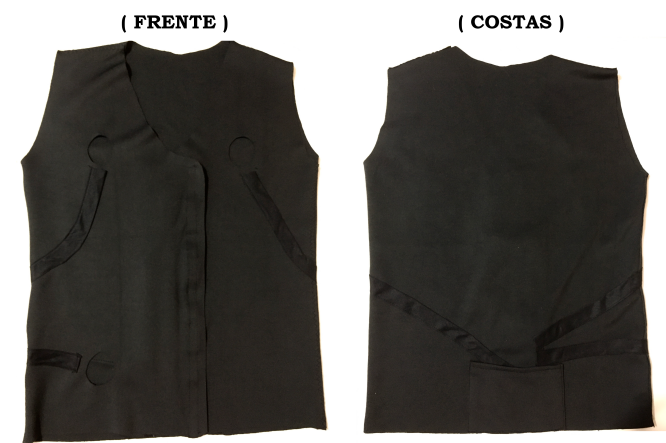

(b) Colete ECG

Figura 2. Detalhamento Técnico e Execução da Interface de Entrada Vestível.

Para embarcar os sensores de captação ECG ao colete, foram criadas pela derivação de membro I, ramificações em tiras de tecido (material em viés de algodão) que direcionassem de forma correta os cabos conectados aos eletrodos. Os sensores de captação do sinal cardíaco utilizados no acoplamento à interface vestível não são de caráter têxtil, pela falta de acesso a malharia de filamentos condutivos específicos para a aquisição e fabricação em laboratórios. Assim, para validar a ideia de aquisição foram utilizados eletrodos de superfície convencionais de prata $(\mathrm{Ag} / \mathrm{AgCl})$, fabricados pela MedPex - Electrodes. Possuem a especificação técnica de ECG para adulto constituídos de espuma de polietileno e gel sólido na face de contato.

Além disso, para garantir a generalidade da peça, no processo de design e modelagem foi construído um bolso de entrada vertical posicionado, a princípio, na face das costas para acoplar o dispositivo. A posição do bolso varia com a modalidade do exame de eletrocardiograma. Se o exame contemplar o ECG de esforço, feito em esteira/bicicleta ergométrica, o bolso se mantém na posição das costas. Se for o exame de ECG de repouso o bolso passa a ser fixo na face frontal ou lateral esquerda da peça.

\subsubsection{Interface de Processamento Analógico: Condicionamento do Sinal Cardíaco}

Como os sinais bioelétricos estão compreendidos na escala de mili a microvolts (sinal cardíaco $1 \mathrm{a} 5 \mathrm{mV}$ ) e, mais facilmente, sujeitos às interferências e ruídos de naturezas distintas, foi utilizado um amplificador de instrumentação. O amplificador de instrumentação adotado para o desenvolvimento do circuito de condicionamento do sinal ECG, é o AD620. Ele apresenta elevada impedância de entrada, baixa resistência de saída, alto CMRR (mínimo 100dB) e baixa tensão de offset na saída. Para minimizar efeitos de interferência RF (Rádio Frequência) foi implementado, em circuito, filtros RC's analógicos, próximos às entradas do amplificador de instrumentação para o sinal de modo comum e para o sinal 
diferencial. Todas essas características e denominações contribuem na redução de ruídos provenientes dos eletrodos no processo de captação do sinal cardíaco.

Foram necessários o projeto e implementação de um filtro ativo anti-aliasing passabaixa de segunda ordem para eliminar sinais e ruídos de altas frequências. Neste caso, o projeto foi instituído para atenuar frequências acima de $200 \mathrm{~Hz}$, preservando a banda de frequência que contempla o sinal cardíaco $(0.1-150 \mathrm{~Hz})$. Essa técnica também evita que haja sobreposição no espectro de frequência ou efeito aliasing, garantindo o teorema de Nyquist, o qual diz que a frequência de amostragem tem que ser no mínimo duas vezes maior que a frequência do sinal amostrado [Floyd 2007].

Além disso, como o microcontrolador não opera com tensões de entradas analógicas negativas, foi necessária a implementação de um circuito de offset capaz de incluir um nível de tensão DC (0.5V) ao sinal cardíaco condicionado pelo AD620. Neste caso, foi utilizado um amplificador operacional configurado no modo somador inversor de tensão, tendo como referência o sinal de $-5 \mathrm{~V}$ fornecido pelo circuito de alimentação com fatores de ganho unitário para cada sinal (FAA_out e Offset) associado à entrada inversora do amplificador somador inversor. Segue na figura 3, o circuito projetado e simulado em ferramenta LtSpice XVII:
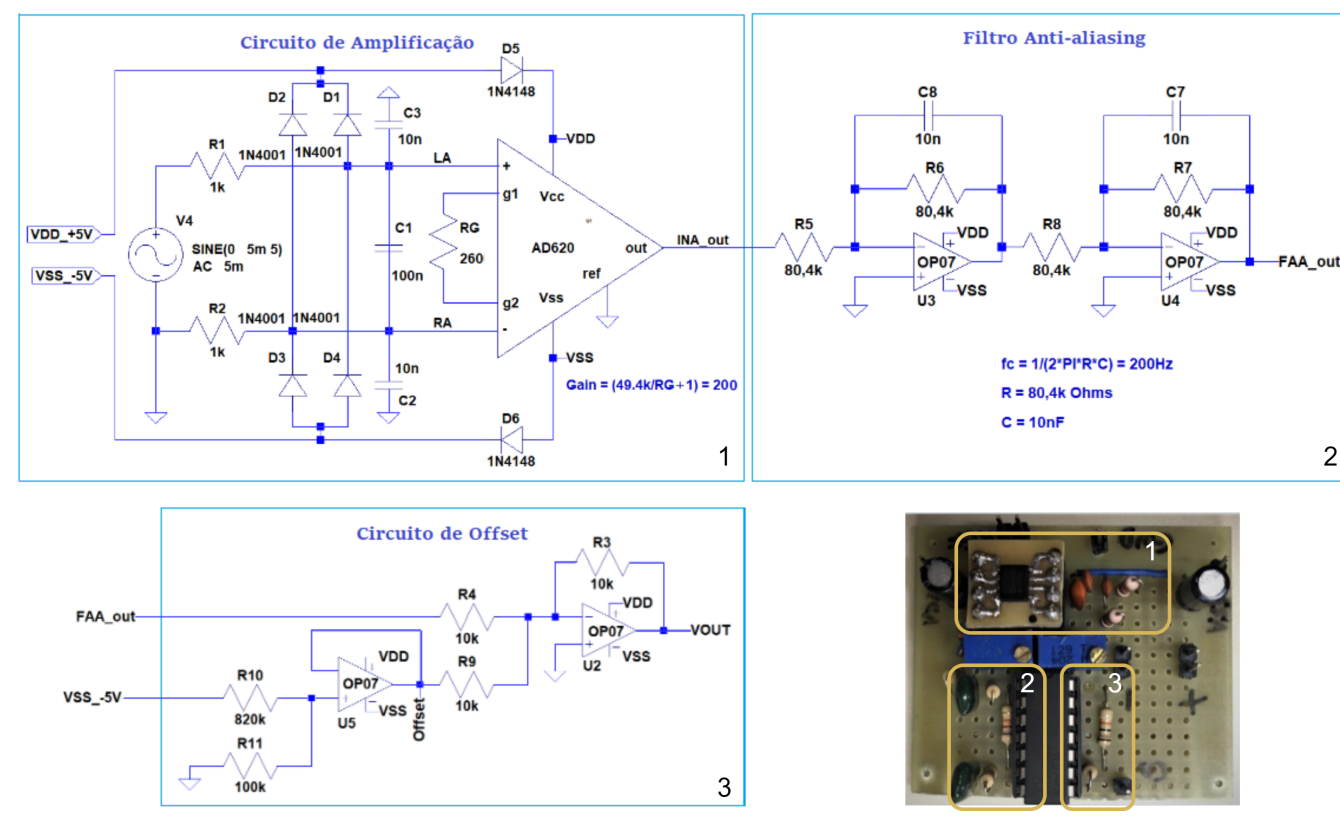

Figura 3. Circuito Completo do Sistema de Condicionamento do Sinal Cardíaco.

\subsubsection{Interface de Processamento Digital: Aquisição e Comunicação sem Fio}

Após o condicionamento do sinal cardíaco faz-se necessário que ele seja processado, armazenado e disponibilizado em uma saída amigável para os profissionais de saúde. O sistema de aquisição é constituído por um conversor analógico-digital (ADC), filtros digitais (passa-baixa, passa-alta e notch), e o sistema de comunicação sem fio é formado por periférico de comunicação WiFi embarcado ao microcontrolador ESP32. 
Operacionalmente, o sinal cardíaco é convertido do domínio analógico para o digital, sendo repassado a um conjunto de filtros digitais, essenciais ao processamento do sinal ECG. Após a etapa de filtragem o sinal é tratado, registrado e o periférico de comunicação WiFi do microcontrolador o transmitirá para a aplicação web contemplada pela interface de saída. A implementação consiste na abordagem de leitura do ADC pelo evento de interrupção do microcontrolador, sendo coletado o valor da entrada analógica a cada $2 \mathrm{~ms}$. Desse modo, o sinal cardíaco foi amostrado a uma frequência de $500 \mathrm{~Hz}$, levando em consideração o teorema de amostragem de Nyquist.

Entendidas as características e técnicas adotadas para o procedimento do uso do ADC, é necessário o entendimento do processo de filtragem do sinal cardíaco. Em processamento digital de sinais, de forma geral, são adotados métodos de filtragens os quais têm por característica selecionar porção do sinal de interesse, bem como eliminar componentes indesejadas do espectro de frequência. Essas técnicas podem ser realizadas tanto em domínio analógico quanto digital. O espectro de frequência do sinal cardíaco para o ECG diagnóstico está compreendido entre 0.1 a $150 \mathrm{~Hz}$ (intervalo variável conforme o domínio da aplicação, por exemplo, para o ECG de monitoramento o espectro seria de 0.5 a $40 \mathrm{~Hz}$ [Webster and Clark 2010]).

Para critério de entendimento das propostas de cada filtro implementado no contexto de aquisição e processamento do sinal cardíaco utilizou-se um filtro passa-alta, que atua na remoção de frequências abaixo de $0.1 \mathrm{~Hz}$, frequências essas geradas pelos potenciais de pele inseridas no contato do eletrodo. Atuam, ainda, para minimizar os efeitos de artefatos de movimentos. Já o filtro passa-baixa, atenua frequências acima de $150 \mathrm{~Hz}$, ou seja, componentes de alta frequência presentes acima do espectro do sinal cardíaco. $\mathrm{O}$ filtro Notch, por sua vez, tem a função de rejeitar componentes de uma frequência específica. Neste caso, utilizou-se para remover ruídos provenientes da fonte de alimentação, tais como o ruído da rede elétrica em $60 \mathrm{~Hz}$, suas harmônicas e demais fontes de ruídos de caráter eletromagnético [Webster and Clark 2010, Clifford 2006].

Os filtros digitais mais conhecidos são os filtros de topologia FIR e IIR. A topologia adotada consiste no filtro IIR (Infinite Impulse Response) denotada por uma filtragem com resposta ao impulso de duração infinita. Ele é implementado de forma recursiva, ou seja, a saída é obtida tanto como uma combinação linear de amostras do sinal de entrada quanto de amostras do sinal das últimas saídas, ou ainda, saídas passadas. Além disso, por apresentar uma realimentação do sinal de saída, possui o diferencial de obter excelente resolução no processo de filtragem com número reduzido de coeficientes e, consequentemente, maior velocidade de processamento. A implementação dos filtros, realizada no microcontrolador, utiliza como base principal o elemento buffer circular: estrutura de dados eficiente no processo de programação orientada à fluxo. O filtro digital IIR implementado no microcontrolador ESP32, pode ser visualizado no Algoritmo 1.

Com os sinais devidamente filtrados é necessário, ainda, que as informações coletadas sejam aparentemente expostas ao usuário final em um Monitor ECG. Para tanto, foi elaborada uma aplicação web que dispõe os dados de forma instantânea. Para que o acesso à página web seja possível o periférico WiFi do microcontrolador foi configurado para operar em modo estação. Logo, o dispositivo passa a funcionar como um cliente wireless, conectado à rede local em que se encontra o módulo. $\mathrm{O}$ acesso é feito por qualquer dispositivo (smartphone ou computador) com capacidade de conexão sem fio. 
Para realizar a troca de dados entre dispositivo e aplicação, é utilizado o protocolo HTTP (Hypertext Transfer Protocol). O sistema web/mobile foi desenvolvido e armazenado no microcontrolador ESP32, por apresentar um sistema de arquivo próprio gerenciável.

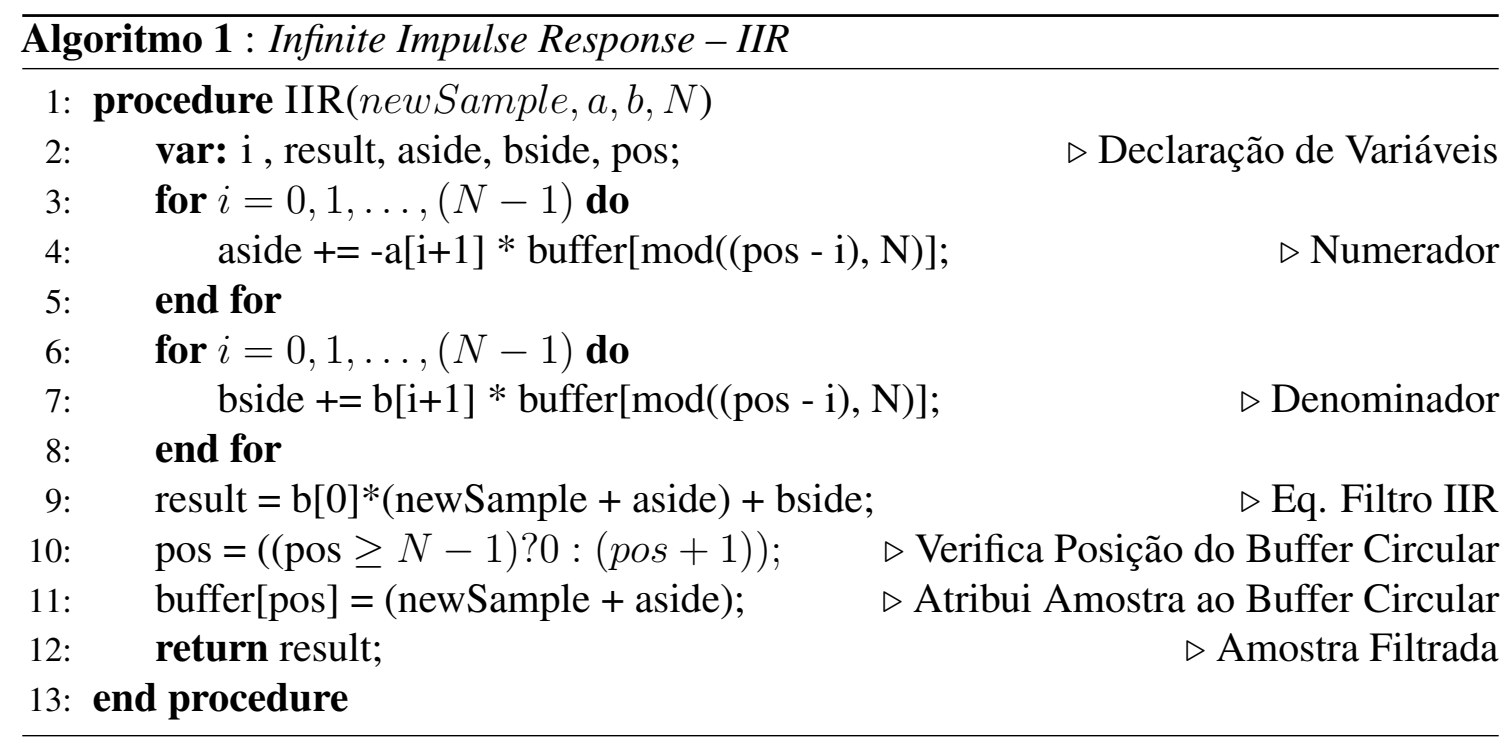

Além disso, na interface de saída composta pela aplicação web/mobile, é possível realizar algumas interações com o gráfico cardíaco de modo a facilitar sua visualização e identificar parâmetros como os batimentos cardíacos, informações sobre o monitoramento da bateria e status de conexão. A aplicação permite também exportar/importar o gráfico cardíaco em formato de imagem, texto e planilha de dados, para que seja armazenado em algum repositório presente no dispositivo de acesso e também enviado, por aplicação, ao email do médico cardiologista previamente cadastrado.

\section{Resultados e Discussões}

Com o término do desenvolvimento do protótipo e tendo em vista todas as partes constituintes do módulo com interface vestível para a aquisição, processamento e transmissão do sinal cardíaco, foi possível passar para as etapas de testes e análises contundentes a fim de verificar os resultados alcançados. Para efeito de validação do condicionamento do sinal cardíaco (processo de amplificação com o AD620), o circuito foi submetido à entrada de um dado bioelétrico real (Indivíduo 1). Os eletrodos embutidos ao colete ECG foram conectados ao corpo, formando a derivação de membro bipolar I, configurando o eletrodo positivo na ramificação do peito esquerdo, o eletrodo negativo na ramificação do peito direito e o eletrodo de referência na parte inferior abdominal à direita. $\mathrm{O}$ resultado adquirido da saída total do circuito de aquisição do sinal cardíaco, após a etapa do filtro anti-aliasing, circuito de offset e aplicação dos filtros digitais é mostrado na figura 4(a).

Com isso, foi possível verificar que o projeto e teste do circuito de condicionamento do sinal ECG satisfazem como o esperado, se comportando de maneira efetiva com o planejado. O amplificador de instrumentação, pelo seu elevado CMRR, rejeitou as tensões de modo comum fornecendo uma saída amplificada do sinal cardíaco. Vale lembrar que, como o sinal cardíaco real varia de uma amplitude de 1 a $5 \mathrm{mV}$ foi obtido uma amplificação do sinal com amplitude próxima de 1.1V (ajustando pelo potenciômetro o ganho do AD620 


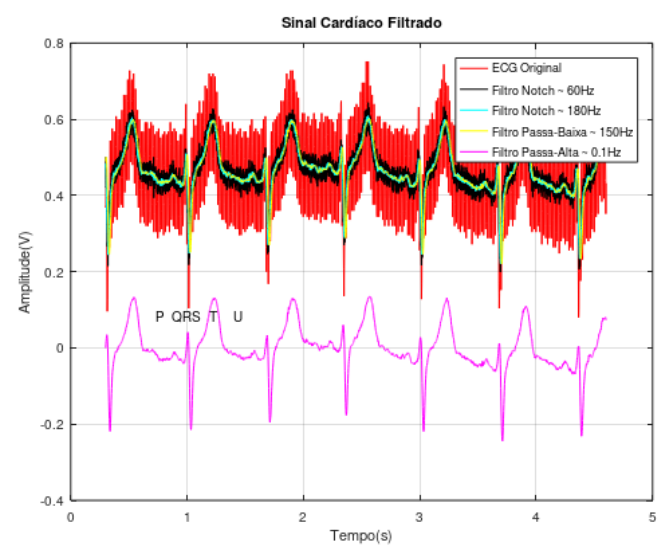

(a) Sinal Cardíaco Filtrado.

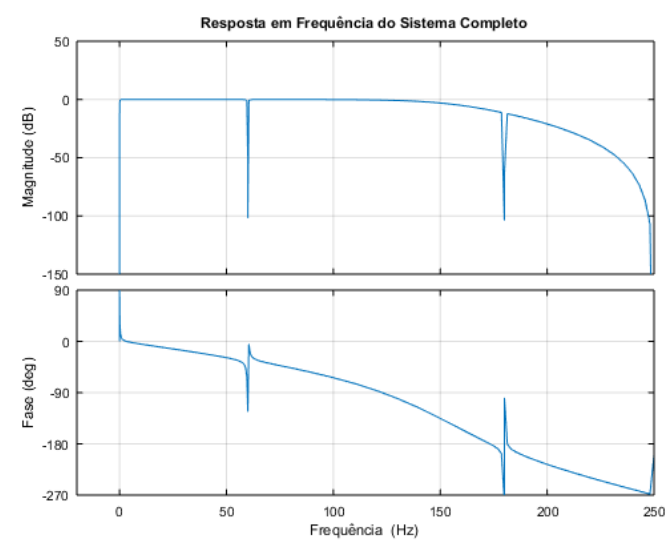

(b) Diagrama de Bode.

Figura 4. Filtros Digitais e Resposta em Frequência.

para aproximadamente $200 \mathrm{~V} / \mathrm{V} \rightarrow R G=260 \Omega$ ). Além disso, foi possível verificar que o circuito de offset funciona como o pretendido (nível médio do sinal em, aproximadamente, $0.5 \mathrm{~V}$ ). A ideia principal é que a tensão de saída do circuito de condicionamento opere dentro do intervalo positivo da tensão de entrada do ADC do microcontrolador.

Para a validação do circuito de aquisição e comunicação sem fio, no que diz respeito o projeto de filtros digitais, implementando os filtros Notch, Passa-Baixa e Passa-Alta (respectivamente) conforme as especificações das frequências identificadas, foi obtido o gráfico cardíaco, com excelente resolução de filtragem. Pela figura 4(b), é possível detectar a resposta em frequência dos três filtros implementados, e em 4(a) o claro reconhecimento da onda P, do complexo QRS e das ondas T e U, características iminentes do sinal ECG. Mostrando que o projeto dos filtros digitais se fez eficaz na remoção de frequências e ruídos indesejados, mantendo a fidelidade do sinal de interesse.
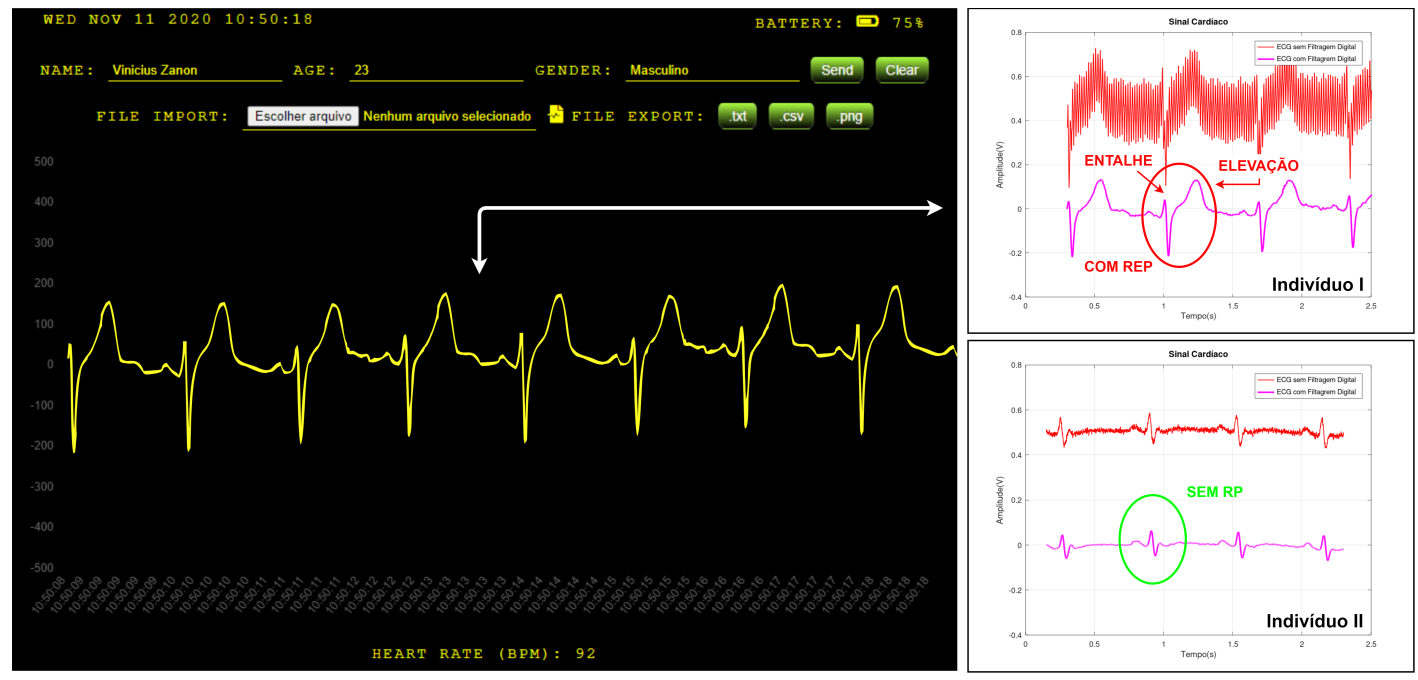

Figura 5. Interface de Saída: Sinais Cardíacos Processados pela Aplicação Web.

Observando a figura 5 é possível verificar o resultado do circuito de condicionamento, aquisição e transmissão do sinal cardíaco submetidos a dois indivíduos diferentes 
já disponibilizado pela Interface de Saída (aplicação web/mobile). O sinal do Indivíduo I apresenta mais ruídos do que o Indivíduo II, justificado pela variante impedância da pele no contato pele-eletrodo, pelo ganho do amplificador ou ainda pela conformidade corpórea. Independente das características de cada indivíduo, os filtros se validam pela remoção do nível DC e artefatos provindos da rede elétrica, sendo possível notar que ambos os indivíduos apresentam ritmos cardíacos regulares.

Ainda nessa etapa de testes, foi observado que o protótipo se fez eficaz na identificação de patologias ou diagnóstico clínico. Em comparação com o Indivíduo II, o Indivíduo I apresenta uma anomalia no sinal, conhecida na cardiologia como Repolarização Precoce Ventricular (RP). A definição padrão de RP é caracterizada por um entalhe no final do complexo QRS, fazendo com que o final não coincida com a linha de base (média do sinal), gerando um segmento ST de concavidade elevada [Aizawa and Kawamura 2019]. A repolarização é comumente observada em jovens atletas que normalmente praticam atividades físicas, sendo não letal à saúde, porém qualquer razão de desconfiança deve-se procurar um médico cardiologista, principalmente se o sinal cardíaco apresentar RP ou inversão da onda T.

\section{Conclusão}

O protótipo vestível para a aquisição, processamento e transmissão do sinal cardíaco sem fio, em exames de eletrocardiograma, apresentou de forma satisfatória os resultados demonstrando a possibilidade de monitoramento remoto via aplicação web. O protótipo apresentou sua prova de conceito validada em placa de ensaio laboratorial, podendo ser reduzido a uma versão final em placa de circuito impresso para fins comercializáveis.

Devido a pandemia de COVID-19, e a consequente impossibilidade de frequentar as dependências da instituição de ensino, não foi possível incluir na interface de entrada a confecção dos eletrodos têxteis. A fabricação seria realizada através de uma malha composta por filamentos de carbono (material condutivo) para a captação do sinal cardíaco sendo possível apenas a validação do módulo com eletrodos comuns de cloreto de prata. Entretanto, ressalta-se que a qualidade da aquisição pela substituição dos eletrodos não comprometeu os resultados do protótipo.

O módulo possui características condizentes na resolução da problemática de eliminação de fios com a base central do eletrocardiógrafo padrão, dado pelo fomento do uso da tecnologia de comunicação sem fio WiFi. Também atua de forma satisfatória no propósito de ser um instrumento de guia metodológico para a execução do exame de eletrocardiograma. Reduzindo os equívocos de execução provenientes do desconhecimento das conexões dos eletrodos e minimizando os efeitos que poderiam ser causados em declarações de diagnósticos equivocados. O dispositivo apresenta potenciais características para ser inserido na prática clínica dos exames de ECG (principalmente o ergométrico), por ser um equipamento sem fio, vestível e confortável, sendo possível imaginar o seu uso, substancialmente, no contexto de pandemia por COVID-19 para o acompanhamento remoto de pacientes.

\section{Referências}

Acar, G., Ozturk, O., and Yapici, M. K. (2018). Wearable Graphene Nanotextile Embedded Smart Armband for Cardiac Monitoring. In 2018 IEEE SENSORS, pages 1-4, New 
Delhi. IEEE.

Acar, G., Ozturk, O., Yapici, M. K., Golparvar, A. J., Elboshra, T. A., and Böhringer, K. (2019). Wearable and Flexible Textile Electrodes for Biopotential Signal Monitoring: A review. In 2019 Electronics, pages 1-25, New Delhi. Electronics.

Aizawa, Y. and Kawamura, A. (2019). Early repolarization in athletes. Journal of Arrhythmia, 35(6):868-869.

Apple (2020). App ECG e notificação de ritmo cardíaco irregular chegam ao Apple Watch.

Carfì, A., Bernabei, R., and Landi, F. (2020). Persistent symptoms in patients after acute covid-19. JAMA - Journal of the American Medical Association, 324(6). p.603-605.

Clifford, G. D. (2006). ECG Statistics, Noise, Artifacts and Missing Data. Artech House, London.

Cohen, J. B., Lotito, M. J., Trivedi, U. K., Denker, M. G., Cohen, D. L., and Townsend, R. R. (2019). Cardiovascular Events and Mortality in White Coat Hypertension. Annals of Internal Medicine, 170(12):853-862.

Fernandes, L. S., Lira, M. C., França, V., Valois, A. A., and Valença, M. P. (2015). Conhecimento Teórico-Prático de Enfermeiras sobre Eletrocardiograma. Revista Baiana de Enfermagem, 29(2). p.98-105.

Floyd, T. L. (2007). Sistemas Digitais: fundamentos e aplicações. Bookman, Porto Alegre, 9 edition.

GE Healthcare (2019). Sistema de patches sem fio Novii.

Heartwear (2019). Monitor cardíaco vestível com inteligência artificial.

Kamišalić, A., Fister, I., Turkanović, M., and Karakatič, S. (2018). Sensors and functionalities of non-invasive wrist-wearable devices: A review. Sensors, 18(6).

Landi, F., Gremese, E., Bernabei, R., and Group, G. (2020). Persistent symptoms in patients after acute covid-19. JAMA - Journal of the American Medical Association, 32(8). p.1613-1620.

Malharia Indaial (1994). Empresa de soluções em tecidos de malhas.

Monteiro, N. O., Marçal, D. C., Bitencourt, I. F., Rocha, L. S., and Dias, E. C. (2018). Habilidades dos Enfermeiros na Realização e Interpretação do Eletrocardiograma em Pronto Atendimento. Revista Científica Univiçosa, 10(1). p.1122-1126.

Pastore, C. A., Pinho, J. A., Samesima, N., Pereira-Filho, H. G., and Kruse, J. C. L. (2016). III Diretrizes da Sociedade Brasileira de Cardiologia sobre Análise e Emissão de Laudos Eletrocardiográficos. Arq Bras Cardiol, 106(4). p.1-23.

Ribeiro, L. P. (2016). Sistema de monitoramento de eletrocardiograma (ecg) utilizando eletrodos secos têxteis.

SBC (2021). Cardiômetro - Mortes por Doenças Cardiovasculares no Brasil.

Wadman, M., Couzin-Frankel, J., and Matacic, C. (2020). A rampage through the body. Science, 368(6489). p.356-360.

Webster, J. G. and Clark, J. W. (2010). Medical Instrumentation - Application and Design. Willey, Danvers, 4 edition. 